\title{
Optimising the assessment of cerebral autoregulation from black box models
}

\author{
N. Angarita-Jaimes ${ }^{1}$, H. Kouchakpour ${ }^{2}$, Jia Liu $^{3}$, R. B. Panerai ${ }^{4}$ and D.M. Simpson ${ }^{2}$ \\ ${ }^{1}$ School of Mechanical Engineering, University of Leeds, Woodhouse Lane, Leeds. LS2 9JT \\ ${ }^{2}$ Institute of Sound and Vibration Research, University of Southampton, Southampton, UK \\ ${ }^{3}$ Shenzhen Institutes of Advanced Technology, Chinese Academy of Sciences, Shenzhen, P. \\ R. China \\ ${ }^{4}$ Department of Cardiovascular Sciences, University of Leicester, United Kingdom \\ E-mail address: $\underline{\text { N.C.AngaritaJaimes@leeds.ac.uk and ds@isvr.soton.ac.uk }}$
}

\begin{abstract}
Cerebral autoregulation (CA) mechanisms maintain blood flow approximately stable despite changes in arterial blood pressure. Mathematical models that characterise this system have been used extensively in the quantitative assessment of function/impairment of CA. Using spontaneous fluctuations in arterial blood pressure (ABP) as input and cerebral blood flow velocity (CBFV) as output, the autoregulatory mechanism can be modelled using linear and nonlinear approaches, from which indexes can be extracted to provide an overall assessment of CA. Previous studies have considered a single - or at most a couple of measures, making it difficult to compare the performance of different CA parameters. We compare the performance of established autoregulatory parameters and propose novel measures. The key objective is to identify which model and index can best distinguish between normal and impaired CA. To this end twenty-six recordings of ABP and CBFV from normocapnia and hypercapnia (which temporarily impairs CA) in 13 healthy adults were analysed. In the absence of a 'gold' standard for the study of dynamic CA, lower inter- and intra-subject variability of the parameters in relation to the difference between normo- and hypercapnia were considered as criteria for identifying improved measures of CA. Significantly improved performance compared to some conventional approaches was achieved, with the simplest method emerging as probably the most promising for future studies.
\end{abstract}

Keywords: blood flow, autoregulation, system identification, modelling.

\section{Introduction}

The active control of the diameter of small cerebral blood vessels protects the brain against 
injury due to insufficient or excessive blood flow following a temporary drop or surge in arterial blood pressure (ABP). This regulatory mechanism is usually referred to as Cerebral Autoregulation (CA). Autoregulation is of great clinical interest as it can be impaired or lost in a number of conditions, such as stroke [1], subarachnoid haemorrhage [2] or head trauma [3]. In much of the published literature, blood flow velocity is recorded by the safe and noninvasive Doppler ultrasound method in response to transient changes in ABP. Sudden deflation of a thigh cuff, large sinusoidal variations in lower-body negative pressure, periodic breathing or squatting, and the Valsalva manoeuvre have all been used to provoke larger changes in ABP [4]. However the most desirable experimental protocol for assessing $\mathrm{CA}$ is to record data from subjects at rest without performing any specific manoeuvres; this is especially relevant for patients during surgery or in intensive care, those highly vulnerable (e.g. neonates), or otherwise unable or unwilling to cooperate. Many recent studies have thus focused on using only spontaneous fluctuations of ABP for the assessment of CA. Even though this approach increases challenges in terms of analysing the recorded signals, and intra and inter-subject variability is high, its effectiveness has been repeatedly demonstrated [4-6].

Algorithms already described in the literature for estimating CA involve system identification (black-box modelling) to represent the relationship between ABP and cerebral blood flow velocity (CBFV). Most of the studies of CA focus on linear methods [5, 7-10] with the more recent inclusion of some nonlinear approaches [8, 10-13]. Although nonlinear techniques can provide improved model fits, their benefit in assessing CA is still unclear with few studies having systematically compared them to linear alternatives.

In the investigation of CA from linear models, parameters extracted from the frequency-, the impulse- or step-response of the models have been used to grade CA. Examples of such autoregulatory parameters include gain, phase and coherence in selected frequency ranges $[5$, $7,8,14]$, or alternatively features of the step-response - e.g. slopes or amplitudes at selected points [9, 14]. An alternative method is provided by the autoregulatory index ARI [15]: this varies from 0 (absent CA) to 9 (excellent CA), with each corresponding to a specific linear filter. The filter that best fits the data determines the ARI for the recording, and this can be applied to spontaneous as well as induced blood pressure variations $[16,17]$. The correlation of the ABP and CBFV time series [18] known as the Mx index, has also been very extensively used (e.g. [19]). The majority of published studies have considered one or two measures of CA for the analysis of cerebral blood flow control from spontaneous variations [10, 14], and no single parameter has become accepted as a gold standard. To date, it is not clear which combination of model and parameter for the assessment of CA is the most appropriate for future research and clinical practice. 
The exploratory work in the current paper aims to compare a range of alternatives and recommend most promising methods. We investigate the performance of both linear and nonlinear models, and compare different measures extracted from the models to assess CA. In the continuing absence of a gold standard measure of CA, the autoregulatory parameters are evaluated on a sample of signals recorded from healthy volunteers in whom temporary impairment of CA was induced by hypercapnia [20,21]. Based on the results, we suggest some promising autoregulatory measures for application in future physiological and clinical studies.

\section{Methods}

\subsection{Data Collection and pre-processing}

The study was performed on 13 healthy volunteer subjects (age $32 \pm 8.8$ years) and was approved by the local Research Ethics Committee. All recordings were made with subjects in the supine position with the head elevated. Middle cerebral artery velocity was measured using a Transcranial Doppler Ultrasound system (Scimed QVL-120) in conjunction with a $2 \mathrm{MHz}$ transducer held in position by an elastic headband. Simultaneously arterial blood pressure (ABP) was non-invasively monitored using a finger cuff device (Ohmeda 2300 Finapress $\mathrm{Bp}$ monitor). End-tidal $\mathrm{CO}_{2}$ (EtCO2) levels were monitored via an infra-red capnograph (Datex Normocap 200). Each recording began with a period of breathing ambient air for approximately 5 minutes, followed by approximately 2 minutes of elevated (EtCO2 ) due to the inhalation of $5 \% \mathrm{CO}_{2}$ in air.

The maximum velocity envelope and the ABP signals were digitised at $200 \mathrm{~Hz}$. After the removal of spikes and other artefacts, the start of each heart cycle was automatically identified (with visual correction) from the ABP sigal, and average ABP and CBFVs were calculated for each heartbeat. This time series was then interpolated with a third-order polynomial, and sampled at a constant rate of $5 \mathrm{~Hz}$.

\subsection{Modelling and Data Analysis}

For each subject a segment of data was selected from before (normocapnia - NC) and during 5\% CO2 breathing (hypercapnia - HC). The former were approx. $300 \mathrm{~s}$ long and the latter 120 s. The initial transients at the onset of $\mathrm{HC}$ was removed until the signals were deemed stable, following visual inspection. The signals were decimated to a new sampling rate of $1 \mathrm{~Hz}$, following anti-alias filtering with a cut-off frequency at $0.5 \mathrm{~Hz}$. These segments of the recordings were normalised by their respective mean values (as used in previous works e.g.[8, $22,23]$ ), and the relative change (expressed in \%) will be denoted by \%ABP and \%CBFV, respectively - see Figure 1. 
For both linear and non-linear models, \%ABP was the input and \%CBFV the output. Linear models were estimated according to the usual least-mean-squares approach. A fifth order (6 coefficient) FIR filter [6] was chosen in order to obtain a parsimonious model which includes the main early part of the autoregulatory response $[6,24]$. The frequency response ('transfer function') was then obtained by applying the Fourier Transform to the impulse response. A $2^{\text {nd }}$ order Volterra-Wiener model, as previously proposed [8, 12, 25], was also estimated using the Wiener-Laguerre (W-L) estimation procedure (for more details see for example [8]). The number of lags used for both the linear and nonlinear kernels was 12 samples (i.e. 12 seconds in duration) and 6 filters were used for the Laguerre expansion.

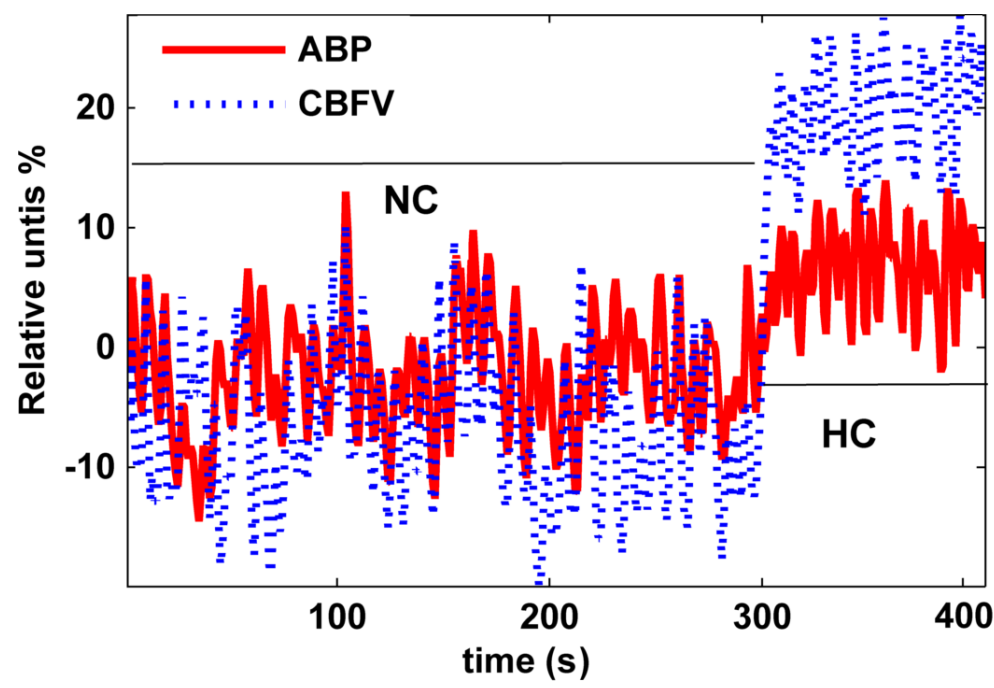

Figure 1. Representative recording of ABP (arterial blood pressure) and CBFV (cerebral blood flow velocity) changes following inhalation of $5 \% \mathrm{CO}_{2}$ in air. The first 300 seconds correspond to normocapnia (NC), and the last segment (120 s) to hypercapnia (HC).

\section{Assessment of Cerebral Autoregulation}

\subsection{Selection of Autoregulatory Parameters}

The final value of the models response (both linear and non linear) after applying an idealised step (see Figure 2A) is a commonly used approach $[6,8,26]$ to assess $\mathrm{CA}$, and will be denoted as FVS (Final Value from the Step); in absent CA, FVS remains elevated [15]. Also, the amplitude at 3 seconds was calculated and expressed as a percentage change from the initial segment of the response (amplitude at 1 second) - denoted PCS (Percentage Change from the Step), as this was shown to be more robust than FVS [6]. 

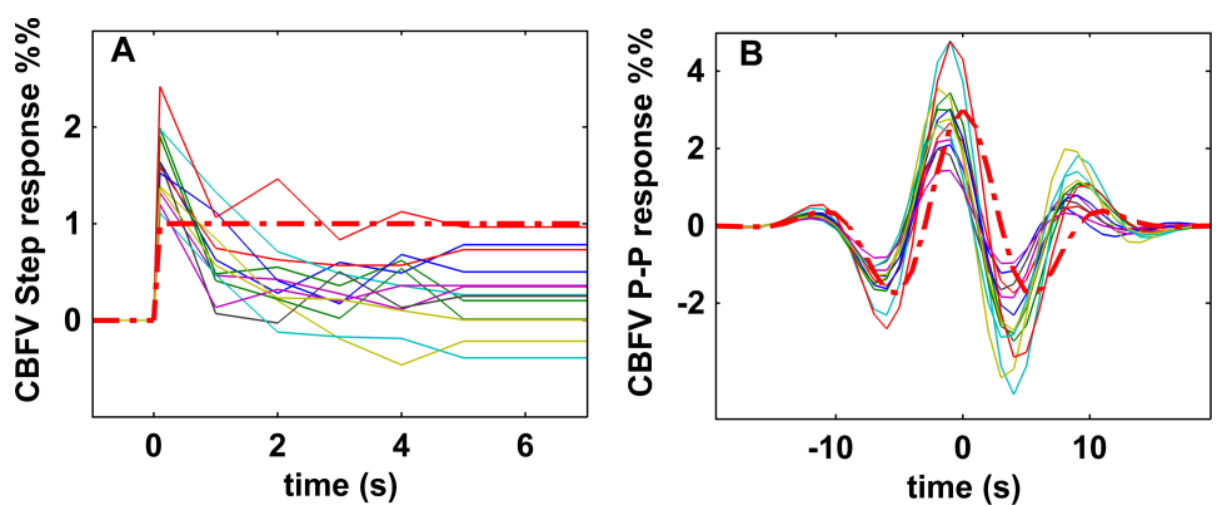

Figure 2. Step (a) and pressure-pulse (b) response for thirteen subjects (solid lines) estimated from a 5 second-long FIR (finite impulse response) model. The inputs are shown as the bold dot-dashed- line.

In [27] a broadband filtered impulse (generated by applying a Gaussian window to a cosine wave at $0.08 \mathrm{~Hz}$ - Figure $2 \mathrm{~B}$ and Figure $3 \mathrm{~A}$ ) was proposed as an alternative test input. This is visually similar to spontaneous fluctuations observed in recorded ABP signals (Figure 3B), and has a centre frequency in the range previously used in the assessment of CA from gain or phase $[5,8,19]$. The response to this input will be denominated as the pressure-pulseresponse (PPR).
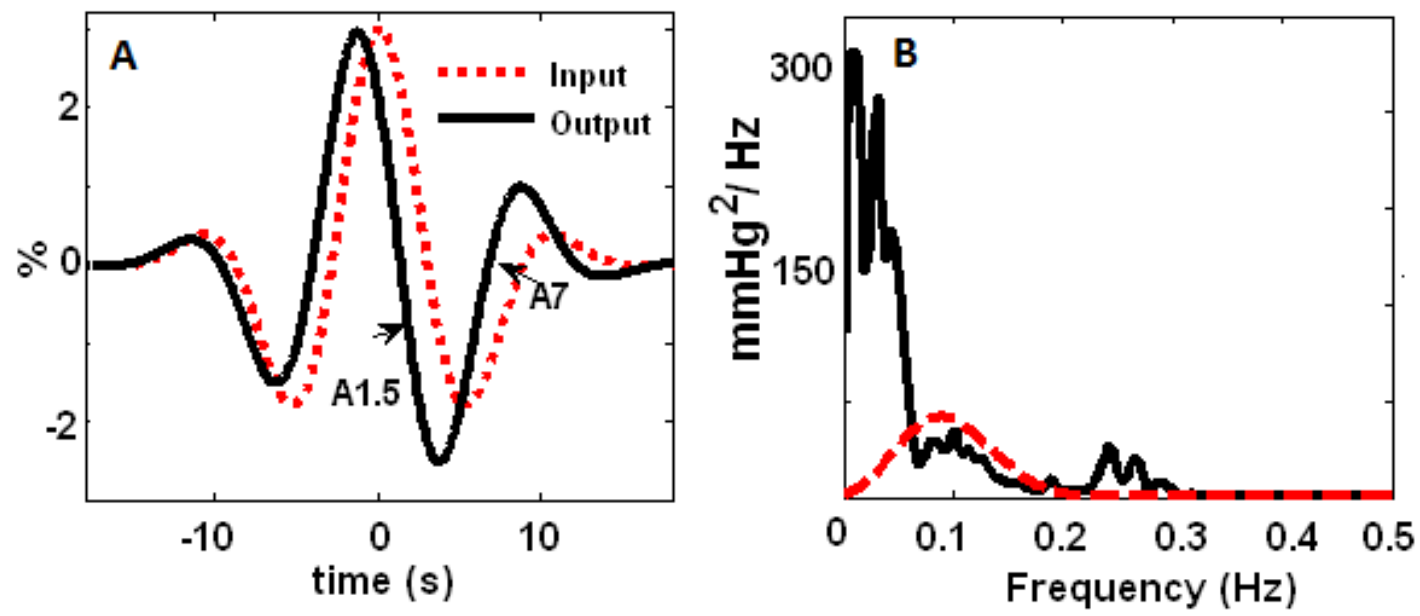

Figure 3. A. The pressure-pulse test input (sinusoid modulated by a Gaussian pulse - dotted line) and an example of the estimated response (\% CBFV cerebral blood flow velocity - solid line). The parameters A1.5 and A7 (amplitudes at 1.5 and 7 seconds respectively) are also shown. B. Autospectra of ABP (arterial blood pressure) for one typical subject (solid line) and of the pressure-pulse input (dotted line). The latter aims to emulate the peak of the former just below $0.1 \mathrm{~Hz}$.

Figure 2B and 3A show the expected left-shift (phase lead) in the PPR [7] resulting from the high-pass characteristics of the autoregulatory response [5, 14]. Results from preliminary work [13] based on simulations using Tiecks model [15] and recorded data showed that the 
PPR at 1.5 seconds (A1.5) and the amplitude at 7 seconds (A7) (as shown in Fig. 3A) provide good separation of different levels of CA and are thus selected for inclusion in the current study.

From transfer function analysis the average phase (Pha) and coherence (Coh) were estimated in the frequency range from $0.07 \mathrm{~Hz}$ to $0.2 \mathrm{~Hz}$ [5, 24]. Using the correlation method [18], $\mathbf{M x}$ was estimated as the average Pearson's correlation coefficient of four equal segments of the $\% \mathrm{ABP}$ and \% CBFV time series. The Autoregulatory Index ARI was calculated by evaluating the set of models proposed in [15] using the parameter values given by the authors. In each recording, the model was applied to the \%ABP signal and the model leading to the highest correlation coefficient between the model generated velocity and the measured \% CBFV determined the ARI. A parametric model based on the coefficients of a first-order (two coefficients) FIR filter [9] was also evaluated; the second coefficient of the filter provided an additional autoregulatory parameter $(\mathbf{H 1})$.

For the nonlinear model, only the parameters extracted from the step response (FVS and PCS) and from PPR (A1.5 and A7) were used.

\subsection{Statistical Analysis}

In order to compare the goodness of fit of different models, the velocity predicted by each model from the recorded ABP data was compared to the measured data and the normalised mean square error (NMSE) was calculated. This is defined as the mean-square value of the difference between the predicted and measured velocity normalised by the mean-square of the measured velocity. Cross-validation was applied on the baseline data, with signals divided into two equal segments of $150 \mathrm{~s}$, one for training and the other for validation and NMSE calculate over the latter. Average values (after swapping the segments) were recorded. Crossvalidation was not applied to the data during hypercapnia, due to the short length of data available in that condition.

Changes in the autoregulatory parameters induced by increased $p \mathrm{CO}_{2}$ were tested using paired $t$-tests. Inter-subject variability was assessed by calculating the standard deviation of the estimated parameters for all subjects during normo- and hypercapnia, and averaging the result; this will be denominated SD. In order to compare the performance of different parameters, whose values are all on quite different scales, SD was normalized by the difference in mean value between $\mathrm{NC}$ and $\mathrm{HC}$. This will be referred to as the normalized standard deviation (SDn).

Estimation errors in CA parameters, reflecting the repeatability or intra-subject variability of measurements, were assessed using Monte Carlo simulations. To this end, 100 simulated signals were generated for each of the recordings. Surrogate \%CBFV signals were generated 
by applying the identified models (linear or non-linear) to the \% ABP signals and then adding random noise to simulate residuals. The noise was modelled based on the residual error in $\% \mathrm{CBFV}$ (i.e. the signal component that cannot be explained by applying the identified models to the \%ABP signals) using an AR model of order 8 selected according to Akaike's information criterion [28]. Then for each of the 100 simulated signals, each of the CA parameters was estimated, and the standard deviation of these gives the intra-subject (within subject) variability. The mean value across the 13 subjects was calculated and also normalized by the parameter's mean difference between normocapnia and hypercapnia (as for SDn) and denoted by $\mathbf{m S D n}$. Low values of SDn and $\mathbf{m S D n}$ thus indicate low dispersion and/or wide separation between groups indicating improved ability to distinguish between normal and impaired CA.

The Shapiro-Wilks test was used to assess normality of all the data sets before applying parametric statistical tests, and subsequently applied parametric and non-parametric statistical test accordingly. To compare the fit of different models we used repeated two-way ANOVA in order to test for the combined effects of modelling techniques and of subjects. We used $k$ repeated samples Friedman tests to evaluate the differences of intra-subject variability (as obtained from Monte Carlo simulations), as these were not normally distributed. Inter-subject variability was evaluated by bootstrap followed by randomization. This approach was tested on simulated data under the null hypothesis, and showed the expected false positive rate.

\section{Results}

Mean CBFVs were significantly different between normo- and hypercapnia ( $p \approx 0.02)$; in all 13 subjects mean $\mathrm{CBFV}$ increased after $\mathrm{CO}_{2}$ inhalation, indicating effective cerebrovascular reactivity and compliance with the protocol.

\subsection{Model Performance}

Table 1 shows the normalised mean-square error in fitting different models to the data.

There was no significant difference between models (ANOVA $p>0.05$ ); pairwise comparisons using the Bonferroni correction for revealed that the fifth order FIR model offered an overall improvement in NMSE compared to the first order FIR model $(p<0.05)$ but not over the W-L model. 


\begin{tabular}{|c|c|ccc|}
\hline \multirow{2}{*}{ Model } & \multicolumn{4}{|c|}{ NMSE \% } \\
\cline { 2 - 5 } & Training & \multicolumn{3}{|c|}{ Validation } \\
\hline FIR - 2 lags & $41 \pm 18$ & 46 & \pm & 21 \\
FIR - 6 lags & $36 \pm 17$ & 44 & \pm & 20 \\
Quadratic W-L & $28 \pm 13$ & 58 & \pm & 27 \\
\hline
\end{tabular}

Table 1. Mean \pm standard deviation NMSE (normalised mean square error) across 13 subjects for the different models applied to the baseline recordings.

\subsection{Assessment of Cerebral Autoregulation}

The different autoregulatory parameters are shown in Table 2 and performance indexes in Figure 4.

\begin{tabular}{|c|c|c|c|c|}
\hline \multirow{2}{*}{ Parameter } & \multicolumn{2}{|c|}{ Normocapnia } & \multicolumn{2}{c|}{ Hypercapnia } \\
\cline { 2 - 5 } & mean & std & mean & std \\
\hline Mx & 0.46 & 0.15 & 0.59 & 0.09 \\
Pha & 0.82 & 0.23 & 0.53 & 0.26 \\
Coh & 0.68 & 0.16 & 0.84 & 0.10 \\
ARI & 5.97 & 1.95 & 4.15 & 2.07 \\
H1 & -1.38 & 0.35 & -0.81 & 0.42 \\
FVS-L & 0.29 & 0.39 & 0.54 & 0.32 \\
FVS - NL & 0.05 & 0.43 & 0.65 & 0.71 \\
PCS-L & 0.76 & 0.17 & 0.55 & 0.12 \\
PCS-NL & 0.84 & 0.27 & 0.49 & 0.19 \\
A1.5-L & -1.10 & 0.70 & -0.16 & 0.39 \\
A1.5-NL & -0.98 & 0.71 & -0.13 & 0.80 \\
A7-L & 0.60 & 0.44 & 0.06 & 0.20 \\
A7-NL & 0.78 & 0.62 & 0.07 & 0.44 \\
\hline
\end{tabular}

Table 2. Autoregulatory parameters: mean and standard deviation from the thirteen subjects.

For all parameters significant differences were found between NC (normocapnia) and HC (hypercapnia) using paired $t$-tests (Figure 4), suggesting that all are able to detect the expected impairment in CA.

\subsubsection{Inter-subject variability}

Autoregulatory parameters (see Figure 4) with small SDn indicate good separation between NC and HC. Amongst the parameters studied A1.5 (linear model - to be denoted as A1.5-L, with similar notation for other parameters), A7-NL (non-linear model), H1 and PCS-L, had the lowest SDn and FVS the highest. Although, the modelling approach used can reduce the inter-subject variability, no significant differences were observed for SDn between linear and 
nonlinear models (ANOVA, $p>0.05$ ). The most notable results from the pairwise randomization tests between all parameters for SDn were that FVS-L was significantly $(p \leq 0.05)$ worse than A1.5-L, A7-L and PCS-L and (at $p \leq 0.1)$ A1.5-L outperformed ARI, A1.5-NL and FVS, and A7-L outperformed A1.5-NL and FVS-L.

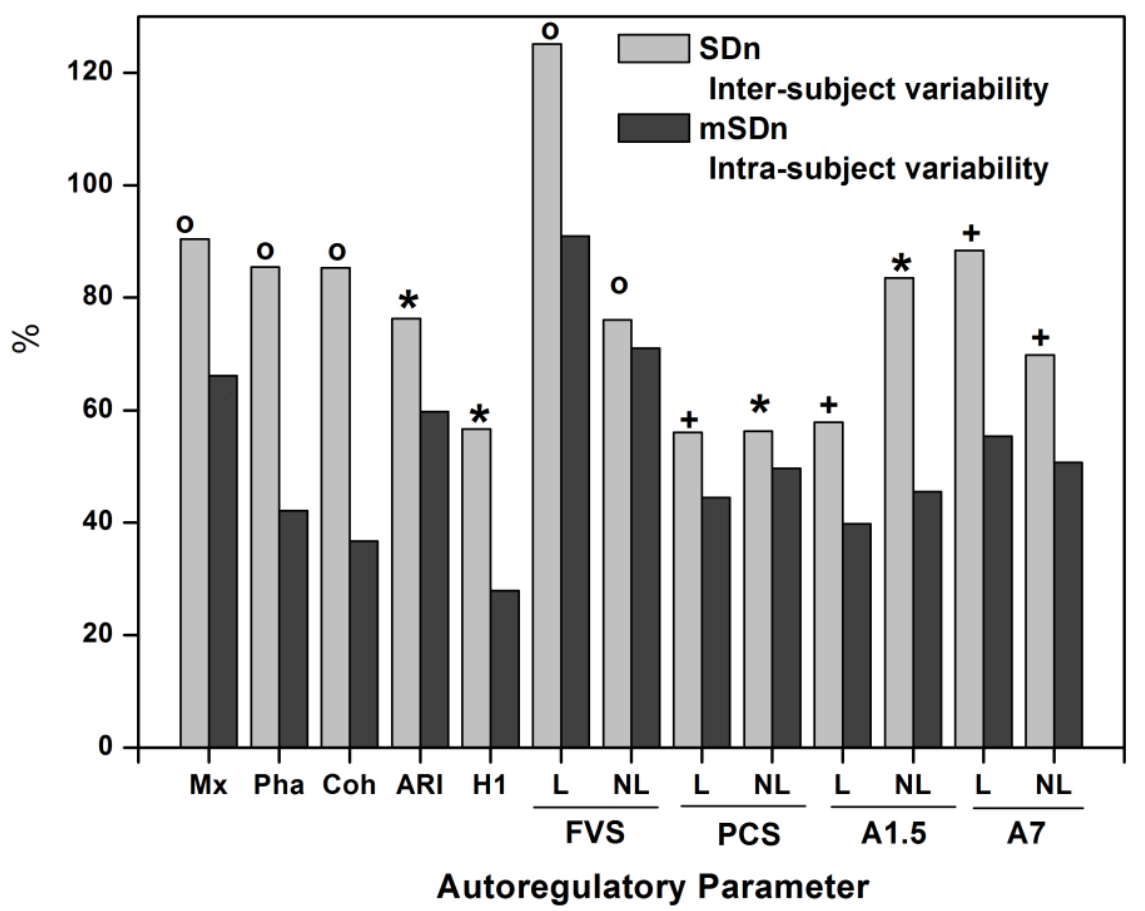

Figure 4. Comparison of the different autoregulatory parameters. L (linear) and NL (non-linear) models respectively. Test of difference between NC (normocapnia and HC (hypercapnia): $+p<0.0005$, $* p<0.005$, o $p<0.05$.

\subsubsection{Intra-subject variability}

Conventional parameters extracted from the frequency response (Mx, Pha, Coh) as well as A1.5-L, A7-L and A7-NL were relatively robust to noise (low mSDn); H1 performed particularly well (Figure 4). There were no significant differences in the variability of estimates between normocapnia and hypercapnia ( $p>0.05$ for all tests).

Friedman test ( $k$-related samples) showed significant differences in the set of thirteen parameters $(p=0.015)$. Post-hoc analysis was also carried out (Wilcoxon signed-ranked test and Bonferroni correction, $p<0.0015$ ), to explore data further. Given the large number of parameters in the test, this was only applied to H1 (which was significant against A7-L, FVSNL and PCS-NL), PCS-L (significant against FVS-NL) and A1.5-L (significant against FVS-NL). 


\section{Discussion}

\subsection{Major Findings}

In this exploratory study, autoregulatory parameters with the lowest intra-subject variability (relative to the difference between NC and HC) are H1, A1.5-L and PCS-L as well as A7(both $\mathbf{L}$ and NL). For inter-subject variability it is A1.5-L, A7-NL, PCS-L and H1 that performed best, in this sample. FVS-L and FVS-NL performed consistently poorly.

A more detailed analysis of the major findings as well as the limitations of the current work follows.

\subsection{Inter-subject variability}

In previous work, the high inter-subject variability of a number of measures of cerebral blood flow control and poor repeatability has been noted $[9,25,29]$. In some of the earlier work [8, $13,25,30]$ the primary concern has been with how well different models fit the data. This however is only an intermediate step in addressing the main challenge: quantifying CA when only spontaneous fluctuations in $\mathrm{ABP}$ and $\mathrm{CBFV}$ are present. The ultimate aim is to extract a single clinically (or physiologically) relevant measure of CA. The results in this work showed that the choice of model and parameters can notably change the variability in the estimates. The current work is probably the most extensive comparison between different parameters of CA calculated from spontaneous variations published to date.

In the continuing absence of a gold-standard measure of CA, we take as criteria for assessing performance the ability to distinguish between normal (during normo-capnia) and impaired (during hypercapnia) CA, in healthy adults. The current work also moves beyond the more established 'test inputs' that give impulse, step or frequency (i.e. sine-wave) responses, by using a 'test input' that is physiologically more realistic, in the form of a broad-band pressure pulse. The results have shown lower dispersion in some of the measures extracted from the pulse response compared to traditional autoregulatory parameters (ARI, Phase, Mx, Coh, FVS), as indicated by SDn and mSDn. Although by the appropriate selection of the autoregulatory parameter the separation between normal and impaired condition can be increased, even for the best performing parameters (H1, A15-L, PCS-L and A7-NL) the SDn (i.e. the ratio of the between subjects standard deviation of parameter, to the mean difference between normocapnia and hypercapnia) is large, at approximately 0.6 and only in a few were some parameters found to outperform others.

\subsection{Intra-subject variability}

The estimated intra-subject variability (mSDn) was notably large compared to the dispersion between subjects (SDn), ranging from 25 to $90 \%$. In the Monte Carlo simulations for the calculation of mSDn added stationary noise was assumed, with time-invariant CA. This is the 
usual assumption made in estimating confidence limits in system identification e.g. [31] but requires further investigation following on from some recent studies on the dynamics of CA $[23,32]$. In the current work the estimated intra-subject variability can however be interpreted as the dispersion of estimates to be expected if the source of errors were only added noise rather than less tractable physiological variation.

Previous studies have shown considerable intra-subject variation for repeated thigh cuff tests [33], repeated recordings with spontaneous fluctuations [22, 25] as well as recordings with controlled breathing where a sliding window was used to estimate short-term variability [25]. Previous analyses that concentrated on the performance of the ARI [25, 32, 33] have shown large variations over time and sudden drops to zero of this index. Although rigorous studies have not been performed to understand the source of this variability, this may be due to the constraint that the Aaslid's model [15] imposes on the index as it only uses a set of ten filters [25]. Temporary absence of autoregulatory responses have also been observed in model-free analysis of spontaneous transients in blood pressure [26]. While such phenomena may reflect genuine temporary absences of CA they could also be the result of spontaneous variations in $\mathrm{CBFV}$ that are unrelated to ABP fluctuations 'masking' autoregulatory responses.

H1 presented the most robust estimate in terms of intra-subject variability, as may be expected following the principle of parsimony, given that it has the smallest number of free parameters in the model. It should be noted that the model fit for this simplest of linear filters was poor in the training set (Table 1), and worse than the fifth order linear model in the validation data. On the other hand, the most sophisticated model tested (W-L) provided the best fit to the training data, but worst performance in the validation data (showing clear evidence of overfitting). However both models provided good measures of CA. It is clear that model fit alone is not a good indicator for performance in assessing CA.

\subsection{Limitations}

There are clearly an almost infinite number of possible parameters that could be used to assess $\mathrm{CA}$, and in the current work only a subset was considered. Given the relatively small sample of recordings available, some caution has to be exercised in interpreting the results, as the relative performance of the methods may reflect peculiarities in the data set available. However, the large differences in performance observed, and statistically significant differences noted probably do indicate which approaches are more promising for further study. Such studies should include repeated recordings of step-wise changes of EtCO2 and longer periods of baseline and in particular hypercapnia, as well as more subjects to allow more robust estimates of the intra- and inter-subject variability. Ideally, one would wish to compare equal-length recordings of normocapnia and hypercapnia, though the lengths used here are compatible with those used in other studies. Shorter data lengths are expected to 
increase intra-subject variability, but in this work, a decrease in standard deviation between NC and HC (table 2), was observed more often (8/13) than an increase (4/13); HC seems to be giving more stable estimates of $\mathrm{CA}$ than $\mathrm{NC}$, for the same length of data.

Within the parameters chosen for analysis here, there are also alternatives that should be considered: here we normalized both $\mathrm{ABP}$ and $\mathrm{CBFV}$ signals by their mean value, as used in some previous works; others have not done so (e.g. [34]), and results may thus be quite different for those CA parameters that are based on amplitude. For those parameters based on signal morphology only (Coh, Ph, ARI, Mx, PCS-L), normalization will not affect the results. For the frequency band for the transfer analysis based parameters (Pha, Coh), we have chosen the 'low-frequency range' $(0.07 \mathrm{~Hz}$ to $0.2 \mathrm{~Hz})$, where others have preferred lower frequency bands (e.g. [22]). As argued in [4], autoregulation is more active at lower frequencies, but also more prone to artefacts; $\mathrm{CO} 2$ in particular has a strong influence at the very low frequencies [12]. Better performance of a given parameter in one frequency band does not imply that it would also be better in a different band. Clearly, similar analysis to that carried out here is required on alternative CA parameters - using a larger dataset to ensure sufficient statistical power in the analysis.

The strong scatter in results may be due not only to noise in the signals and differences among subjects but also the influence of other physiological variables such as $p \mathrm{CO}_{2}, p \mathrm{O}_{2}$, cerebral metabolic rate, and intracranial and venous pressure which were not analysed here. Even though studies including multivariate models have been published [30, 35], so far there has been little analysis as to whether this type of models may offer an improvement in terms of separation between normal and impaired conditions compared to the univariate approaches. This issue needs to be addressed in further studies.

In the absence of a gold-standard for measuring $\mathrm{CA}$, a comparison was made between normocapnia (with assumed active CA) and hypercapnia (inducing temporary impairment). Other studies e.g. [36] have used patient outcome as a measure in comparing methods to assess CA. The use of $\mathrm{CO} 2$ as a means to temporarily induce impaired $\mathrm{CA}$ is open to questioning. Clearly, the parameters considered all respond to hypercapnia, and in a manner that would be expected from the usual concept of autoregulation. However, given the complexity of the mechanisms controlling cerebral blood flow [21], one cannot be certain whether these parameters directly reflect autoregulation or some (related) physiological phenomenon. Furthermore it is possible that different clinical conditions (or $\mathrm{CO}_{2}$ levels) have distinct effects [1] and that a single measure of CA may not be optimal for all applications [37]. Throughout this paper we have assumed (as does most of the cited literature) that the parameters used measure cerebral autoregulation, However, given the complexity of the physiology and the often poor correlation found between parameters that have all been 
considered to measure CA ([38]), they may not all reflect identical phenomena of cerebrovascular control. In the current paper we have expanded on previous work, but remained firmly within the premise of most of these studies, by comparing the performance of different individual CA parameters based on the established protocol of normocapnia and hypercapnia, and exploiting spontaneous variability in signals. Further work to refine the concept of autoregulation, improve understanding of its physiology and link these to clinically feasible and robust measurement methods, is still required.

\section{Conclusions}

Among the parameters of CA examined, the amplitude of the pressure pulse response (PPR) A1.5 (linear model) and A7-( $\mathbf{L}$ and NL)showed the clearest distinctions between the different levels of CA. However, the results obtained from H1 suggest that this parameter, estimated from a very simple model shows the smallest normalized intra-subject variability (estimation error - mSDn) with good inter-subject variability (SDn), may be the method of choice. This method also lends itself well to the analysis of relatively short data segments and thus would be best suited for further studies of time-varying (adaptive) estimates of dynamics in cerebral blood flow control.

\section{Acknowledgements}

We would like to thank Drs. Stephanie Foster and Lingke Fan and Prof. David Evans (Leicester Royal Infirmary, UK) for generously providing the anonymized data and Innovation China UK and EPSRC for funding support. We also wish to thank the anonymous reviewers for constructive comments that have helped to improve the paper.

Competing interests: None declared

Funding: Innovation China UK and EPSRC

Ethical approval: Not required

\section{References}

1. Dawson, S.L., M.J. Blake, R.B. Panerai, and J.F. Potter, Dynamic but not static cerebral autoregulation is impaired in acute ischaemic stroke. Cerebrovascular Diseases, 2000. 10(2): p. 126-132.

2. Giller, C.A., The Frequency-Dependent Behavior of Cerebral Autoregulation. Neurosurgery, 1990. 27(3): p. 362-368.

3. Czosnyka, M., P. Smielewski, P. Kirkpatrick, D.K. Menon, and J.D. Pickard, Monitoring of cerebral autoregulation in head-injured patients. Stroke, 1996. 27(10): p. $1829-1834$. 
4. Panerai, R.B., Assessment of cerebral pressure autoregulation in humans - a review of measurement methods. Physiological Measurement, 1998. 19(3): p. 305-338.

5. Zhang, R., J.H. Zukerman, C.A. Giller, and B.D. Levine, Transfer function analysis of dynamic cerebral autoregulation in humans. American Journal of Physiology-Heart and Circulatory Physiology, 1998. 274(1): p. H233-H241.

6. Liu, J., D.M. Simpson, and R. Allen, High spontaneous fluctuation in arterial blood pressure improves the assessment of cerebral autoregulation. Physiological Measurement, 2005. 26(5): p. 725-741.

7. Birch, A.A., M.J. Dirnhuber, R. Hartley-Davies, F. Iannotti, and G. Neil-Dwyer, Assessment of autoregulation by means of periodic changes in blood pressure. Stroke, 1995. 26(5): p. 834-7.

8. Panerai, R.B., S.L. Dawson, and J.F. Potter, Linear and nonlinear analysis of human dynamic cerebral autoregulation. American Journal of Physiology-Heart and Circulatory Physiology, 1999. 277(3): p. H1089-H1099.

9. Simpson, D.M., R.B. Panerai, D.H. Evans, and A.R. Naylor, A Parametric Approach to Measuring Cerebral Blood Flow Autoregulation from Spontaneous Variations in Blood Pressure. Annals of Biomedical Engineering, 2001. 29(1): p. 18-25.

10. Panerai, R.B., M. Chacon, R. Pereira, and D.H. Evans, Neural network modelling of dynamic cerebral autoregulation: assessment and comparison with established methods. Medical Engineering \& Physics, 2004. 26(1): p. 43-52.

11. Mitsis, G.D., P.N. Ainslie, M.J. Poulin, P.A. Robbins, and V.Z. Marmarelis, Nonlinear modeling of the dynamic effects of arterial pressure and blood gas variations on cerebral blood flow in healthy humans. Post-Genomic Perspectives in Modeling and Control of Breathing, 2004. 551: p. 259-265.

12. Mitsis, G.D., R. Zhang, B.D. Levine, and V.Z. Marmarelis, Modeling of nonlinear physiological systems with fast and slow dynamics. II. Application to cerebral autoregulation. Annals of Biomedical Engineering, 2002. 30(4): p. 555-565.

13. Angarita-Jaimes, N. and D.M. Simpson, Optimizing the Assessment of Cerebral Autoregulation from Linear and Nonlinear Models. BIOSIGNALS 2011 Proceedings of the International Conference on Bio-inspired Systems and Signal Processing, Rome, Italy, 26-29 January, 2011, 2011.

14. Liu, Y., A.A. Birch, and R. Allen, Dynamic cerebral autoregulation assessment using an ARX model: comparative study using step response and phase shift analysis. Medical Engineering \& Physics, 2003. 25(8): p. 647-653.

15. Tiecks, F.P., A.M. Lam, R. Aaslid, and D.W. Newell, Comparison of Static and Dynamic Cerebral Autoregulation Measurements. Stroke, 1995. 26(6): p. 1014-1019.

16. Panerai, R.B., R.P. White, H.S. Markus, and D.H. Evans, Grading of cerebral dynamic autoregulation from spontaneous fluctuations in arterial blood pressure. Stroke, 1998. 29(11): p. 2341-2346.

17. Simpson, D.M., R.B. Panerai, E.G. Ramos, J.M.A. Lopes, M.N.V. Marinatto, J. Nadal, and D.H. Evans, Assessing blood flow control through a bootstrap method. Ieee Transactions on Biomedical Engineering, 2004. 51(7): p. 1284-1286.

18. Piechnik, S.K., X. Yang, M. Czosnyka, P. Smielewski, S.H. Fletcher, A.L. Jones, and J.D. Pickard, The continuous assessment of cerebrovascular reactivity: A validation of the method in healthy volunteers. Anesthesia and Analgesia, 1999. 89(4): p. 944949.

19. Reinhard, M., Cerebral Autoregulation in Carotid Artery Occlusive Disease Assessed From Spontaneous Blood Pressure Fluctuations by the Correlation Coefficient Index. Stroke, 2003. 34(9): p. 2138-2144. 
20. Aaslid, R., K.F. Lindegaard, W. Sorteberg, and H. Nornes, Cerebral autoregulation dynamics in humans. Stroke, 1989. 20(1): p. 45-52.

21. Paulson, O.B., S. Strandgaard, and L. Edvinsson, Cerebral Autoregulation. Cerebrovascular and Brain Metabolism Reviews, 1990. 2(2): p. 161-192.

22. Gommer, E.D., E. Shijaku, W.H. Mess, and J.P. Reulen, Dynamic cerebral autoregulation: different signal processing methods without influence on results and reproducibility. Med Biol Eng Comput, 2010. 48(12): p. 1243-50.

23. Liu, J., M.D. Simpson, J. Yan, and R. Allen, Tracking time-varying cerebral autoregulation in response to changes in respiratory PaCO2. Physiol Meas, 2010. 31(10): p. 1291-307.

24. Panerai, R.B., J.M. Rennie, A.W.R. Kelsall, and D.H. Evans, Frequency-domain analysis of cerebral autoregulation from spontaneous fluctuations in arterial blood pressure. Medical \& Biological Engineering \& Computing, 1998. 36(3): p. 315-322.

25. Panerai, R.B., P.J. Eames, and J.F. Potter, Variability of time-domain indices of dynamic cerebral autoregulation. Physiological Measurement, 2003. 24(2): p. 367381.

26. Panerai, R.B., B.J. Carey, and J.F. Potter, Short-term variability of cerebral blood flow velocity responses to arterial blood pressure transients. Ultrasound in Medicine and Biology, 2003. 29(1): p. 31-38.

27. Simpson, D.M.a.B., A.A, Optimizing the assessment of autoregulation from black-box models. 4th IET International Conference on Advances in Medical, Signal and Information Processing (MEDSIP 2008). , 2008.

28. Marple, S.L., Digital spectral analysis : with applications. Prentice-Hall signal processing series. 1987, Englewood Cliffs, N.J.: Prentice-Hall. xx, 492 p.

29. Brodie, F.G., E.R. Atkins, T.G. Robinson, and R.B. Panerai, Reliability of dynamic cerebral autoregulation measurement using spontaneous fluctuations in blood pressure. Clinical Science, 2009. 116(5-6): p. 513-520.

30. Mitsis, G.D., M.J. Poulin, P.A. Robbins, and V.Z. Marmarelis, Nonlinear modeling of the dynamic effects of arterial pressure and CO2 variations on cerebral blood flow in healthy humans. Ieee Transactions on Biomedical Engineering, 2004. 51(11): p. 19321943.

31. Ljung, L., System identification: theory for the user. 1987: Prentice Hall, Englewood Cliffs, NJ.

32. Panerai, R.B., M. Moody, P.J. Eames, and J.F. Potter, Dynamic cerebral autoregulation during brain activation paradigms. American Journal of PhysiologyHeart and Circulatory Physiology, 2005. 289(3): p. H1202-H1208.

33. Mahony, P.J., R.B. Panerai, S.T. Deverson, P.D. Hayes, and D.H. Evans, Assessment of the thigh cuff technique for measurement of dynamic cerebral autoregulation. Stroke, 2000. 31(2): p. 476-480.

34. Claassen, J.A., B.D. Levine, and R. Zhang, Dynamic cerebral autoregulation during repeated squat-stand maneuvers. J Appl Physiol (1985), 2009. 106(1): p. 153-60.

35. Panerai, R.B., D.M. Simpson, S.T. Deverson, P. Mahony, P. Hayes, and D.H. Evans, Multivariate dynamic analysis of cerebral blood flow regulation in humans. Ieee Transactions on Biomedical Engineering, 2000. 47(3): p. 419-423.

36. Czosnyka, M., P. Smielewski, A. Lavinio, J.D. Pickard, and R. Panerai, An assessment of dynamic autoregulation from spontaneous fluctuations of cerebral blood flow velocity: a comparison of two models, index of autoregulation and mean flow index. Anesthesia and Analgesia, 2008. 106(1): p. 234-9, table of contents. 
37. Panerai, R.B., Cerebral Autoregulation: From Models to Clinical Applications. Cardiovascular Engineering, 2007. 8(1): p. 42-59.

38. Tzeng, Y.C., P.N. Ainslie, W.H. Cooke, K.C. Peebles, C.K. Willie, B.A. MacRae, J.D. Smirl, H.M. Horsman, and C.A. Rickards, Assessment of cerebral autoregulation: the quandary of quantification. Am J Physiol Heart Circ Physiol, 2012. 303(6): p. H658-71. 